\title{
Jamming for a 2D granular material $\dagger$
}

\author{
Jie Zhang, † T. S. Majmudar, $§$ M. Sperlథ and R. P. Behringer* \\ Received 6th January 2010, Accepted 19th May 2010 \\ First published as an Advance Article on the web 2nd June 2010 \\ DOI: $10.1039 / \mathrm{c000147c}$
}

This paper focuses on the nature of jamming, as seen in two-dimensional frictional granular systems consisting of photoelastic particles. The photoelastic technique is unique at this time, in its capability to provide detailed particle-scale information on forces and kinematic quantities such as particle displacements and rotations. These experiments first explore isotropic stress states near point $\mathrm{J}$ through measurements of the mean contact number per particle, $Z$, and the pressure, $P$ as functions of the packing fraction, $\phi$. In this case, the experiments show some but not all aspects of jamming, as expected on the basis of simulations and models that typically assume conservative, hence frictionless, forces between particles. Specifically, there is a rapid growth in $Z$, at a reasonable $\phi$ which we identify with as $\phi_{c}$. It is possible to fit $Z$ and $P$, to power law expressions in $\phi-\phi_{c}$ above $\phi_{c}$, and to obtain exponents that are in agreement with simulations and models. However, the experiments differ from theory on several points, as typified by the rounding that is observed in $Z$ and $P$ near $\phi_{c}$. The application of shear to these same 2D granular systems leads to phenomena that are qualitatively different from the standard picture of jamming. In particular, there is a range of packing fractions below $\phi_{c}$, where the application of shear strain at constant $\phi$ leads to jammed stress-anisotropic states, i.e. they have a nonzero shear stress, $\tau$. The application of shear strain to an initially isotropically compressed (hence jammed) state, does not lead to an unjammed state per se. Rather, shear strain at constant $\phi$ first leads to an increase of both $\tau$ and $P$. Additional strain leads to a succession of jammed states interspersed with relatively localized failures of the force network leading to other stress-anisotropic states that are jammed at typically somewhat lower stress. The locus of jammed states requires a state space that involves not only $\phi$ and $\tau$, but also $P$. $P, \tau$, and $Z$ are all hysteretic functions of shear strain for fixed $\phi$. However, we find that both $P$ and $\tau$ are roughly linear functions of $Z$ for strains large enough to jam the system. This implies that these shear-jammed states satisfy a Coulomb like-relation, $|\tau|=\mu P$.

\section{Introduction}

The collection of papers in this special issue testifies to the intense interest in jamming of disordered systems, such as dense granular materials, that are far from equilibrium. The absence of thermodynamic equilibrium for granular systems in particular, has been at the heart of an effort to develop new kinds of statistical models, some of which we explore here. ${ }^{1-4} \mathrm{~A}$ great challenge is to account for their rich structure, which involves filamentary force networks ${ }^{5-12}$ as in Fig. 1. A key part of the focus on these systems arises from the proposal by Liu and $\mathrm{Nagel}^{13}$ of a universal jamming diagram for a broad range of systems that includes foams, colloids, molecular glasses and granular materials, among others. We sketch this diagram in the left part of Fig. 2. The idea is that there is a region of low temperature (if that applies), low shear stress, and high density, where a material is jammed, i.e. mechanically stable. Density is expressed in terms of the packing fraction, $\phi$, or its inverse in Fig. 2 (left), which is defined as the

Department of Physics, Duke University, Durham, NC, USA. E-mail: bob@phy.duke.edu

$\dagger$ This paper is part of a Soft Matter themed issue on Granular and jammed materials. Guest editors: Andrea Liu and Sidney Nagel.

† Current address: Los Alamos National Laboratory, USA

$\S$ Current address: New York University, NY, USA

\ Current address: DLR, Cologne, Germany fraction of the system volume (area in two dimensions) that is occupied by solid material. There have been many studies that have focused on point $\mathbf{J}$ in this diagram for the case of zero or low shear stress. An important goal of the present studies is to better understand the role played by shear stress, an issue that has been relatively unexplored from the point of view of jamming. However, the role of shear stress has been of interest in the context of soil and geomechanics for a considerable time. ${ }^{14} \mathrm{~A}$ key issue is the failure of granular systems which are under a load that includes both isotropic stress (e.g. pressure, $\mathrm{P}$ ) and also shear

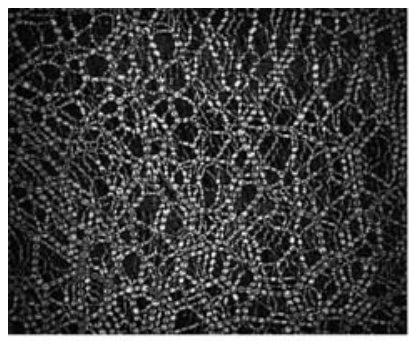

(a)

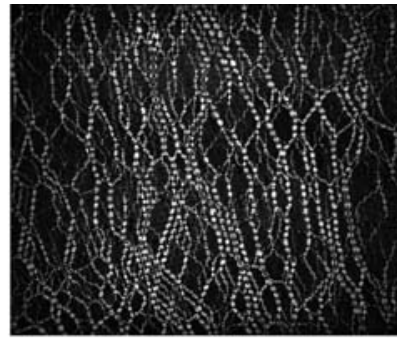

(b)
Fig. 1 Photoelastic images for isotropic (a) and anisotropic (b) states. We discuss below the experimental techniques used to generate these images. 

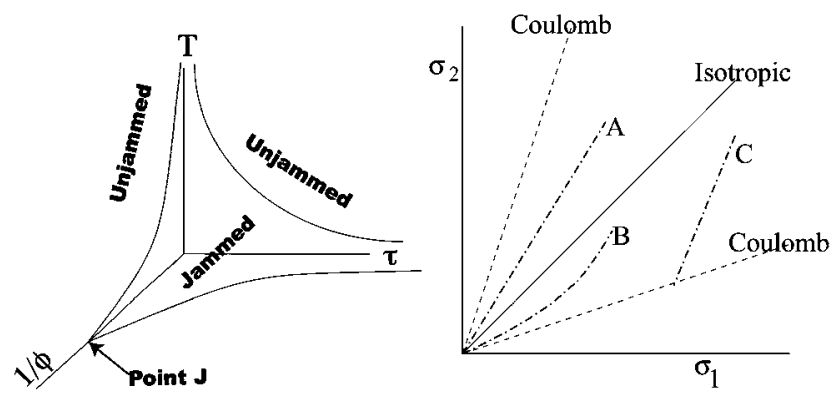

Fig. 2 Left: Representation of jamming diagram, after Liu and Nagel. The three axes are the temperature, which applies in certain systems (e.g. molecular glasses), inverse packing fraction ( $\phi$ is the volume/area occupied by solid for 3D/2D systems), and $\tau$ is the shear stress. Right: Schematic of a Coulomb picture for mechanically stable states for a granular material for two dimensions, in terms of the principal stresses, $\sigma_{i}$. The dashed lines are the failure loci where $|\tau|=\mu P$. Inside this cone, the material is mechanically stable. From this perspective, point $J$ is at the origin.

stress, $\tau$. When the shear stress exceeds a critical threshold relative to the pressure, such a system fails. This type of criterion was the basis of Coulomb's famous work of over two centuries ago. ${ }^{15}$ The basic form of this scenario is sketched in the right part of Fig. 2 for the two-dimensional case. The $\sigma_{i}$ are the principal stresses, i.e., the eigenvalues of the shear stress tensor, $\sigma$, and states within the Coulomb cone denoted by the dashed lines are mechanically stable. Note that the pressure is given by $P=\left(\sigma_{1}+\right.$ $\left.\sigma_{2}\right) / 2$ and the shear stress by $\tau=\left(\sigma_{2}-\sigma_{1}\right) / 2$. If the failure threshold (for a noncohesive material) is $\tau= \pm \mu P$, where $\mu$ is a constant, then the corresponding failure loci in the $\sigma_{1}-\sigma_{2}$ plane are given by the dashed lines $\sigma_{2}=[(1 \pm \mu) /(1 \mp \mu)] \sigma_{1}$. Note that the density of the system does not appear explicitly here (although it is incorporated in models such as Critical State Soil Mechanics ${ }^{14}$ ), but rather the important entities are stresses. The isotropic jammed states, $\tau=0$, lie along the dotted line. Paths in stress space starting within the jammed (mechanically stable) region, such as $\mathrm{A}, \mathrm{B}$, or $\mathrm{C}$, lead to failure when they reach the Coulomb cone. There are a number of interesting cases here, including paths such as A, B, or the isotropic line which fail at zero stress, and path $\mathrm{C}$ which fails under finite stress. In the LiuNagel picture, all three paths would originate in the jammed region, with $T=0$, and densities above the value at point J. Paths $\mathrm{A}$ and $\mathrm{B}$ would approach the boundary of the jammed region at point $\mathbf{J}$, whereas path $\mathrm{C}$ would intersect the jammed region at a point with $\tau \neq 0$.

Below, we review recent experimental tests ${ }^{8,12,16}$ carried out by the present authors that used photoelastic particles to test models for isotropic jamming near point $\mathbf{J}$ and to probe the role of stress anisotropy on jamming. From these studies, several important conclusions follow. First, under isotropic conditions, granular materials do, by and large, exhibit behavior near point $\mathbf{J}$ that is consistent with expectations from numerical simulations ${ }^{16-20}$ (MD/DEM) and on theoretical constructs based on generalized statistical formulations. ${ }^{1-4}$ The latter were first proposed by Edwards et $a .^{1}{ }^{1}$ and have been more recently developed by Snoeijer et al., ${ }^{2}$ Tighe et $a .^{21}$ and Henkes et al. ${ }^{3,4}$ Second, under anisotropic stress conditions, careful accounting must be taken of not only the density and shear stress, but also the pressure. In particular, there exist densities below that for isotropic jamming, which can support jammed stress-anisotropic states. ${ }^{12}$ Hence, the $T=0$ jamming picture for granular materials must account for shear stress, density and pressure.

We note that there are some clear differences in isotropic and anisotropic cases at a heuristic level, as seen in images of the force chain structures, Fig. 1. We also see at a more microscopic level, that the distributions of normal contact forces differ between isotropic and anisotropic states, ${ }^{8}$ as seen in Fig. 3.

In the remainder of this work, we first briefly describe our experimental approach, which uses photoelastic particles contained in a two-dimensional (2D) biaxial device. The 'biax' allows us to prepare states over a range of stress conditions, and the use of photoelastic particles allows us to make detailed quantitative measurements of forces, displacements and rotations. We then review observations for stresses and contact numbers, $Z$, for a $2 \mathrm{D}$ granular system, first under isotropic conditions $^{16}$ near point $\mathrm{J}$, and then for strongly anisotropic states $^{12}$ that are produced by applying shear strain to an isotropically unjammed state. Hence, for the latter case, the density is below the isotropic jamming value. Such states imply the existence of a jamming diagram that involves not only shear stress and packing fraction, but also pressure as an independent variable. We present concluding remarks and a hypothetical jamming diagram in the final section.

\section{Experimental techniques}

As noted above, for the experiments discussed in this work, we used a 2D biax. Fig. 4 (top) illustrates this apparatus, with which we can deform a given rectangular sample of particles into any other desired rectangular shape, within the limits of the apparatus. Of particular interest is the case of pure shear (compression in one direction, equal dilation in the other, and fixed overall area), and isotropic compression (equal compression in both directions).

The particles used in these studies are disks manufactured from photoelastic material. When a photoelastic material is subject to stress, it becomes birefringent, and when viewed between crossed polarizers, it exhibits light and dark bands, as in the bottom left image of Fig. 4. For circular polarizers, the transmitted light along a ray traversing a $2 \mathrm{D}$ section is given by

$$
I=I_{o} \sin ^{2}\left[\left(\sigma_{2}-\sigma_{1}\right) C t / \lambda\right]
$$

where $I_{o}$ is the incident intensity of light with wavelength, $\lambda$, and where $C$ is the stress optic coefficient. Hence, the bands encode the detailed stress within each particle. For a granular system, these stresses are generated by the (vector) forces at the contacts on each particle. Previous researchers have used photoelasticity to probe granular systems, ${ }^{5-7}$ but our approach is unique in that we use photoelasticity to obtain the contact forces for large collections of particles. For more details, the reader may consult. ${ }^{8,11,12,22,23}$ The basic idea is that we carry out a nonlinear least-squares fit of the photoelastic image for each particle to the known solution for the photoelastic response within a particle due to point contact forces. In turn, this solution is based on the stress fields given by linear elasticity theory for point contacts 

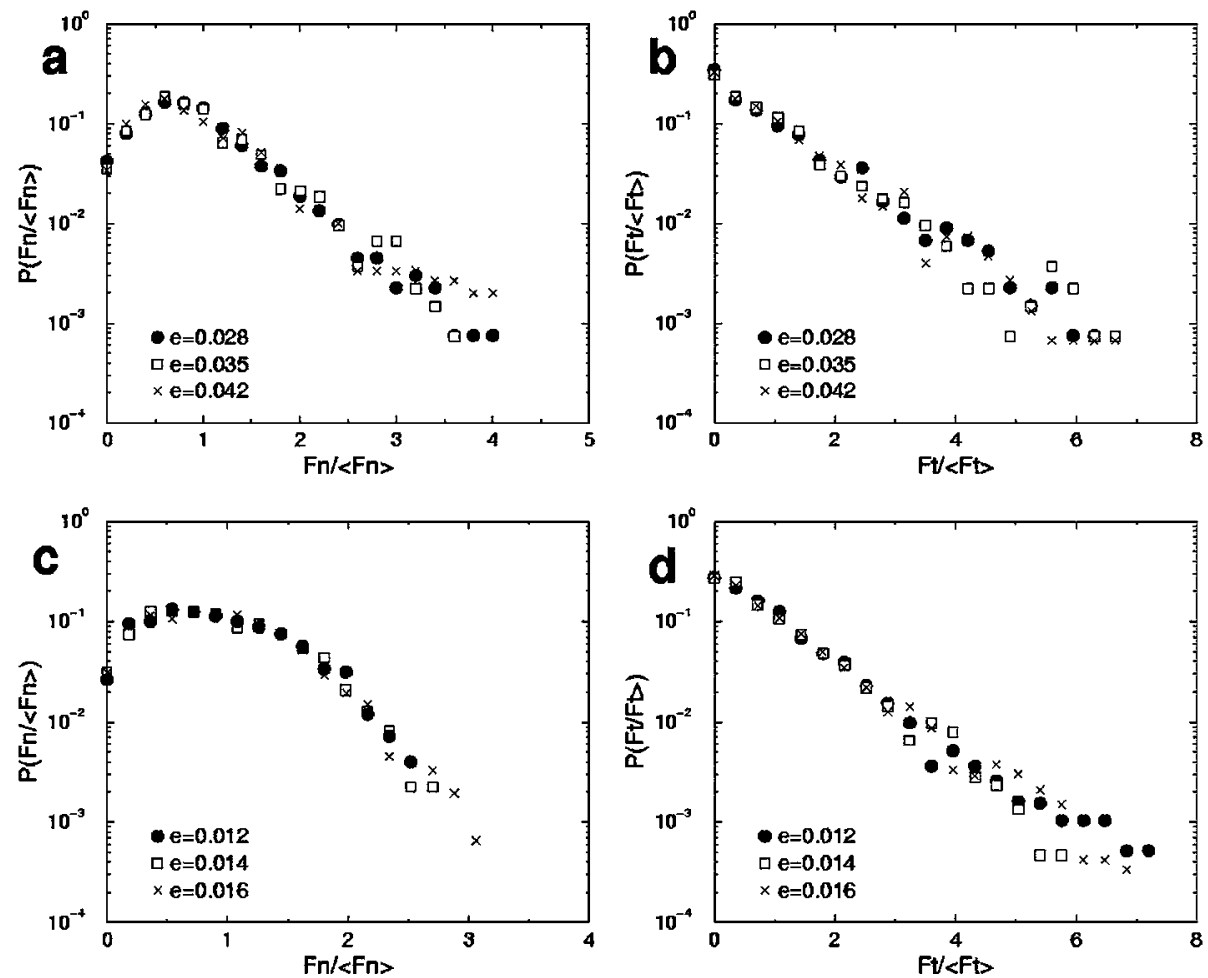

Fig. 3 Distributions for the normal, $F_{n}$, and tangential, $F_{t}$, interparticle contact forces. Parts a and b pertain to a stress-anisotropic state, such as part b of Fig. 2. Parts c and d pertain to a stress-isotropic state, such as part a of Fig. 1.

acting on a disk. This provides the photoelastic response through eqn (1). In this procedure, the fit parameters are the vector contact forces on a particle from its neighbors. To demonstrate the reasonableness of this approach, we contrast an original color photoelastic image and the image produced using the fitdetermined forces in Fig. 5. Note that the photoelastic response, as given by eqn (1), is sensitive to the color/wavelength of the incident light. We take the approach of using white incident light, and color filter the resulting images. The righthand image of Fig. 5 is derived from a color filtered version of the lefthand image. Of course, we must also determine the location of the particles, and the inter-particle contacts. In the process, we also track the rotation and displacement of individual particles as the system is slowly deformed. The rotational motion of the particles is tracked by means of a thin bar drawn on each particle with fluorescent ink. Under ordinary light, the bars are nearly invisible, and do not interfere with the photoelastic measurements. However, they glow brightly under UV light, Fig. 4 (bottom, right). Hence, our most general experimental procedure for measuring particle properties requires three separate images, as typified by the blow-ups in Fig. 4: one with polarizers for the photoelasticity measurements, one under ordinary light without polarizers for identifying particle centers, and a third taken under UV illumination and without polarizers to determining particle rotation.

From the determination of the contact forces, we obtain as an immediate consequence the distributions of the contact forces, $P(F)$. Since the forces are vectoral, it is useful, as in Fig. 3, to separately consider the normal and the tangential components, $F_{n}$ and $F_{t}$ respectively, where the latter are due to friction.
We use several approaches for characterizing the force and contact networks. Since these need not be isotropic, it is important to maintain full tensoral characterizations. The force anisotropy is evident in Fig. 1(b) and 9. In particular, the contact network becomes manifestly anisotropic during cyclic shear. A simple geometric measure of the network is then the fabric tensor, $R_{i j}$ :

$$
R_{i j}=\frac{1}{N} \sum_{k=1}^{N} \sum_{c=1}^{c_{k}} n_{i k}^{c} n_{j k}^{c}
$$

The summation and $N$ include only non-rattler disks, $c_{k}$ is the number of contacts on disk $k$, and $n_{l k}{ }^{c}$ is the $l$ th component of the unit branch vector pointing from the center of the disk $k$ to a contact $c$. The definitions of relevant quantities are illustrated in Fig. 6. We consider a rattler disk to have less than two detectable contacts. The average contact number, $Z$, is then given by the trace of the fabric tensor $R_{i j}$. Here, we do not extensively explore the geometric anisotropy, but the interested reader can obtain more information elsewhere ${ }^{12}$ for the present system regarding this issue.

Additionally, we consider the stress tensor $\sigma_{i j}$ and the force moment tensor, $\hat{\sigma}_{i j}$. These quantities reflect the anisotropy of the force networks. The local force moment tensor is

$$
\hat{\sigma}_{i j}=\sum_{c=1}^{c_{k}} f_{i k}^{c} r_{j k}^{c}
$$

and the system-averaged stress tensor is

$$
\sigma_{i j}=\frac{1}{A} \sum_{k=1}^{N} \hat{\sigma}_{i j} .
$$




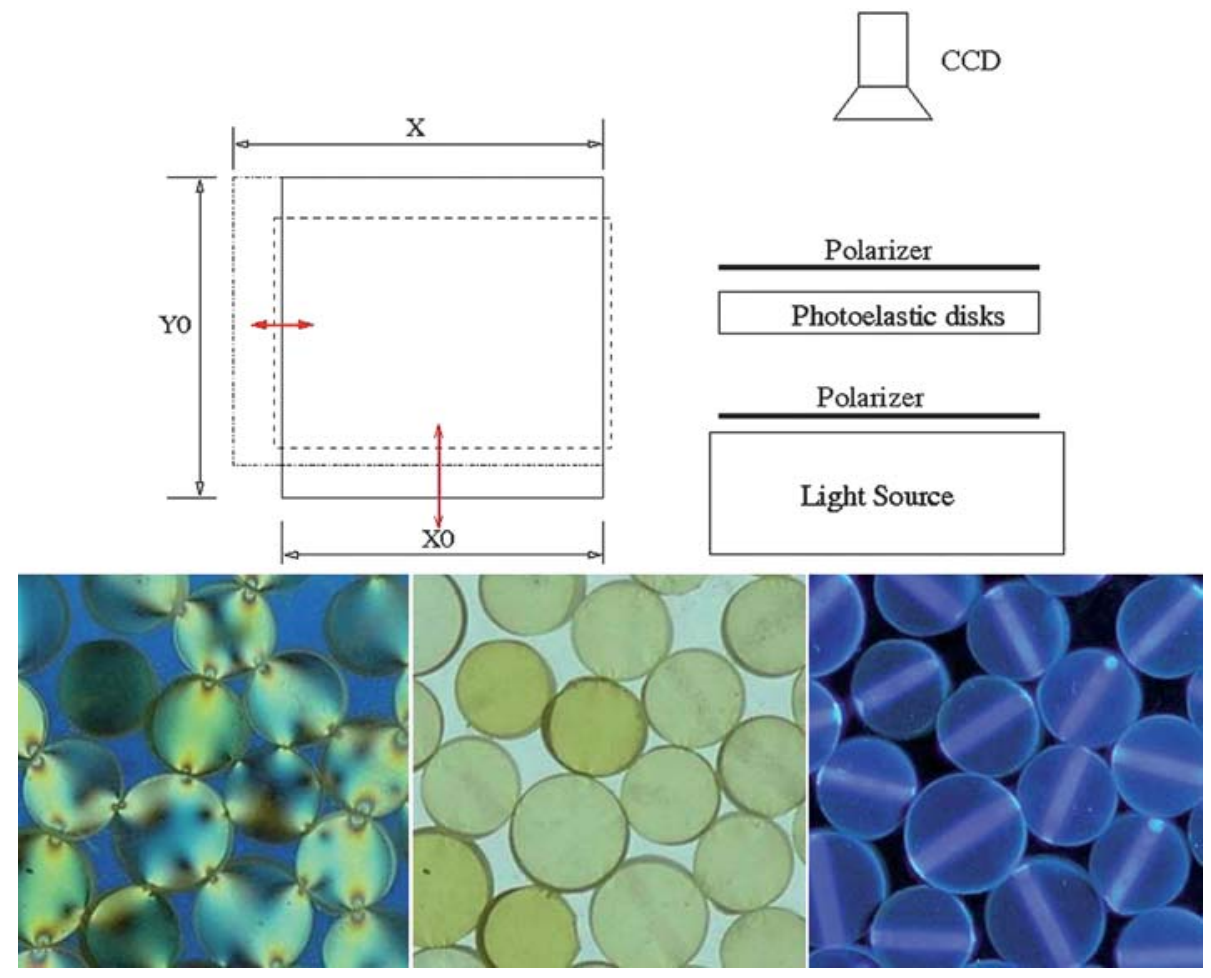

Fig. 4 Sketch of apparatus (top row) and small sections of the three image types that we obtain. Top row: Left: top view of the biaxial apparatus (biax), a 2D 'biax'. Pairs of opposing boundaries in the $x$ and $y$ directions move under precise computer control to produce desired quasi-static strains. These boundaries confine photoelastic particles which also lie on a smooth slippery sheet of Plexiglas. Right: Sketch showing a side view to indicate the photoelastic imaging process. A camera is mounted above the biax, and the whole apparatus, including the disks, are sandwiched between crossed circular polarizers. Following each small strain step, we obtain three images, as shown in the bottom row: one with crossed polarizers (left), one without polarizers (center), and one without polarizers but with UV illumination (right).
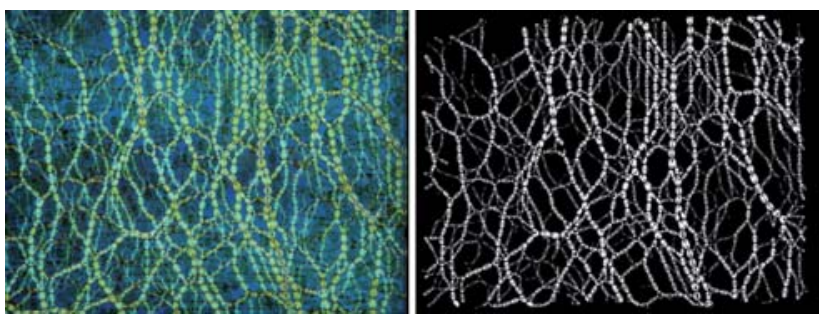

Fig. 5 Original color photoelastic image (left) and the image produced by the contact forces computed by the force-inverse algorithm for a colorfiltered version of this image (right).

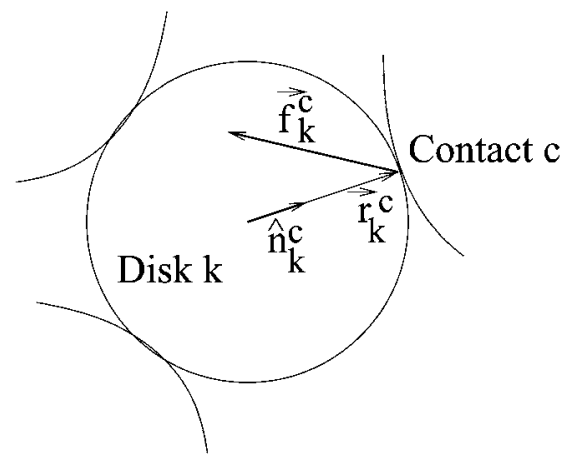

Fig. 6 Sketch explaining the notation for definitions of the fabric, forcemoment and stress tensors.
$A$ is the system area; $N, c_{k}, i, r_{j k}^{c}$ and $j$ are the same as in the expression of $R_{i j}$ (e.g. Fig. 6); $f_{i k}{ }^{c}$ is the $i$ th component of the contact force on particle $k$ at contact $c$. The sum and difference of $\sigma_{1}$ and $\sigma_{2}$, the eigenvalues of $\sigma$, divided by 2, yield $P$ and $\tau$, as defined in the introduction. Below, we take $\sigma_{1} \leq \sigma_{2}$, so that $\tau \geq 0$.

We note one final experimental issue which is important in determining $Z$, and to a lesser extent, $\sigma$, for states very near jamming. The most sensitive and accurate way to determine contacts is to look for a photoelastic response from particles that are in apparent contact. ${ }^{16}$ However, when the stresses are small, the photoelastic response at some contacts may fall below our detection threshold. Nevertheless, we can correct for missed contacts very close to jamming. As discussed below, we find that the distribution of normal contact forces scales with the mean normal force, $\left\langle F_{n}\right\rangle$.

$$
P\left(F_{n}\right)=\left\langle F_{n}\right\rangle^{-1} f\left(F_{n} /\left\langle F_{n}\right\rangle\right),
$$

where $f$ is to a reasonable approximation, the same function for all mean forces. We can use this fact to estimate the number of the missed contacts reasonably well. Forces below our measurement threshold also affect the stresses, but to a smaller extent. We expect that we miss a fraction of contacts given by

$$
\int_{0 c}^{F} P\left(F_{n}\right) d F_{n} / \int_{0}^{\infty} P\left(F_{n}\right) d F_{n},
$$


where $F_{c}$ is a small known cut-off force below which we cannot detect the photoelastic response. For the particles used here, this force is roughly the weight of a particle. Hence, the measured $Z$ 's are lower than their true values by

$$
\int_{F c}^{\infty} P\left(F_{n}\right) d F_{n} / \int_{0}^{\infty} P\left(F_{n}\right) d F_{n} .
$$

We also underestimate the pressure by a factor of

$$
\int_{F c}^{\infty} F_{n} P\left(F_{n}\right) d F_{n} / \int_{0}^{\infty} F_{n} P\left(F_{n}\right) d F_{n} .
$$

To simplify the calculation, this last expression assumes that all particles have the same radius, which is a reasonably good assumption. The resulting correction in $Z$ is $\sim 15 \%$ very near jamming, but negligible once $Z$ is slightly bit above 3.0. Due to the fact that the stresses depend linearly on the contact forces, corrections for missed contacts for these quantities are significantly smaller, only $1-2 \%$ close to the jamming transition, and entirely negligible above jamming. We consider these corrections in data below, where to simplify the correction, we assume that the force distribution is an exponential.

\subsection{Experimental procedures for compression/decompression: Isotropic case}

The experimental approaches are somewhat different depending on whether we are considering isotropic or aniostropic systems. For the isotropic case, we probed the region near point $\mathbf{J}$ using two different protocols. In the first protocol, we gradually compressed the system from an initially stress-free state, taking small strain steps until the system was above the jamming density. In the second approach, we decompressed the system, again by small strain steps, starting in an already compressed/ jammed isotropic state. The end state was a (nearly) stress-free state. The results for both protocols are similar above $\phi_{c}$; however, for densities below jamming, the data for $Z$ obtained by compression are a few percent below those for decompression. In what follows, we show data obtained by the second approach. After each decompression step, we apply tapping to relax stress in the system. We conclude that the preparation protocol does have an effect, and that other methods might produce somewhat different results. The approach used here is designed to produce as low-energy state as possible, which is roughly analogous to the annealing process invoked in some simulations. ${ }^{19}$ After each decompression, we obtain two images, with and without polarizers, respectively. The former is used to compute contact forces and eventually stresses. The latter is used to obtain the disk centers. In this case, we did not track particle rotations.

For the data presented below, we performed two sets of experiments. The first experiment was carried out using a larger range, $0.8390 \leq \phi \leq 0.8650$, and a correspondingly larger step size, $\Delta \phi=0.016$. After we had identified the jamming region, we carried out a second set of experiments at a finer scale with $0.840745 \leq \phi \leq 0.853312$, and with a finer step size, $\Delta \phi=$ 0.000324 .

\subsection{Experimental procedure for pure shear: anisotropic case}

The experiments considered here probe the evolution of a system under pure shear, where the density $\phi=0.795 \pm 0.003$, is lower than what is required to obtain a jammed isotropic state. Pure shear consists of compression in one direction, the ' $y$-direction' and a corresponding dilation in the $\mathrm{x}$-direction, such that the total system area remains fixed. We start with an initially square set of boundaries that are filled with 1568 bidisperse (so as to avoid crystallization) photoelastic disks. The sample consists of roughly $80 \%$ particles of diameter $\approx 0.74 \mathrm{~cm}$, and $20 \%$ particles of diameter $\approx 0.86 \mathrm{~cm}$. We determined the area mass density by two independent measurements, from which we then obtain $\phi$ from the photoelastic material mass density. We estimate that the resulting $\phi$ 's are accurate to \pm 0.003 .

We prepare the initial state as closely as possible to isotropic and stress-free. We then shear the system by compression and dilation along the two independent axes, as in Fig. 4 (top-left), keeping the system area constant. We characterize the deformation in terms of the strain, $\epsilon$, along the $x$-axis with $\epsilon=\left(x-x_{0}\right) /$ $x_{0}$. Here, $x_{0}$ is the initial size of the square. After reaching a maximum deformation $\epsilon_{\max }$, we reverse the shear by compression along the $x$-axis and expansion along the $y$-axis until the system boundaries have reached their initial square configuration. We then continue to shear in the reverse direction, (In other experiments, ${ }^{12}$ we have also carried out cyclic shear, but here we chiefly focus on only one shear cycle.) We carry out this process in small incremental quasi-static steps such that $|\epsilon|$ changes by $\delta \epsilon= \pm 3.3 \times 10^{-3}$. After each step, we stop and acquire three sets of images, as typified by Fig. 4; blow-ups from such figures are shown in the bottom row of Fig. 4. Thus, we obtain an image with polarizers in place (left-most image), without the polarizers (middle) and under UV light (right) so as to determine the rotation of the particles.

\section{Experimental results}

\subsection{Jamming for the isotropic case}

The point of the isotropic studies was two-fold. First, we sought to test recent simulations and theory ${ }^{16-20,24}$ which indicate that near jamming, collections of spherical particles, should exhibit a discontinuous increase in $Z$, at a critical volume fraction, $\phi_{c}$. Below $\phi_{c}, Z$ and $P$ are expected to be 0 . At $\phi_{c}, Z$ is expect to jump discontinuously, and above $\phi_{c}, Z$ and $P$ are predicted to increase as power laws in $\phi-\phi_{c}$. The exponent $\beta$ for $Z-Z_{c}$ is expected to be $1 / 2$ (or nearly $\mathrm{so}^{20}$ ), and the exponent, $\psi=\alpha_{f}-1$, for $P$ is expected to depend on the inter-particle contact force, which in turn is parameterized by the exponent, $\alpha_{f}$. We note that the systems studied theoretically were typically frictionless, and subjected to isotropic overall strains. In view of the second point, the shear stress was $\tau=0$.

Second, we sought to test recent predictions, based on novel entropy approaches (i.e. the force/stress ensemble) by Henkes et $a .^{3,4}$ These predictions, which are a first attempt at constructing a field theory of the transition at point $\mathbf{J}$ led to the particular $P-Z$ relation, involve a generalized 'temperature'-like variable, with both $Z$ and $P$ given as specific functions of this quantity. We are able to test these predictions by determining the relation between $P$ and $Z$ and observing whether the data are consistent with the Henkes et al. prediction.

As noted above, the models of interest typically involve frictionless particles, whereas the experiments are inherently 
frictional. The presence or absence of friction affects $Z_{c}$, and perhaps other aspects of the jamming transition. For instance, in the isostatic limit, $Z$ is 4 for frictionless disks, whereas for frictional disks, $Z$ is around and slightly above 3 , for friction coefficients that are typical of many physical grains. ${ }^{25}$ Predictions such as those for critical exponents, amplitudes, etc. may also need modification. Nevertheless, one might expect that the systems of frictional particles and systems of frictionless particles would exhibit similar behavior, if the frictional forces are typically small relative to the normal forces. In recent experiments, we have found that statistically this is true, and that in the mean, the typical inter-grain frictional forces are $\sim 10 \%$ of the normal forces. $^{8}$

We now turn to experimental data for $Z$ and $P$. In the inset of Fig. 7, we show data for $Z$ over a broad range of $\phi$. Here, we show data both with rattlers (stars) and without rattlers (squares). Specifically, the average $Z$ can be computed either by counting only the force bearing disks, or by counting all the disks including rattlers which do not contribute to the mechanical stability of the system. Here, we consider as rattlers, all the disks which have less than two observable contacts. The data show a significant, but not discontinuous, rise in $Z$ at the transition density corresponding to $\phi_{c} \simeq 0.84$, indicating the onset of system-wide jamming. We note that below, we apply corrections to data for $Z$ obtained from a sheared system. The correction in this case is possible because we have sufficient data to be

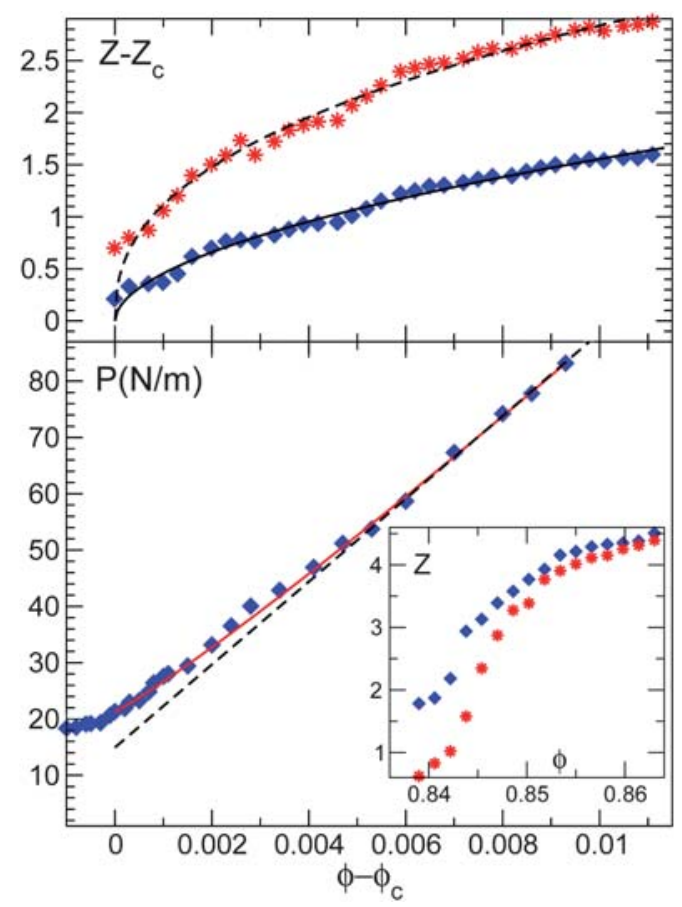

Fig. 7 Data for the average contact number and pressure at the jamming transition. Top and bottom panels show $Z-Z_{c}$ and $P$ vs. $\phi-\phi_{c}$, respectively. Different symbols indicate rattlers included (stars) or excluded (diamonds). Dashed and full curves in the top panel are powerlaw fits $\left(\phi-\phi_{c}\right)^{\beta}$ with $\beta=0.495$ and 0.561 (with and without rattlers, respectively). Lower panel: Full curve gives the fit $\left(\phi-\phi_{c}\right)^{\psi}$ with $\psi=1.1$; dashed line shows a linear law for comparison. Inset: $Z v s$. $\phi$ over a larger range in $\phi$. confident that $P(F)$ is constant regardless of the shear strain. However, the data for the isotropic case has not been corrected because we do not have enough data to be sure that $P(F)$ is invariant with strain in the compressive case.

Above this $\phi$, the variations of $P(\phi)$ with/without rattlers tend to merge, although at lower $\phi$, they differ. Data for $P(\phi)$ in Fig. 7 remain at a nearly flat background below jamming, and then grow above $\phi_{c}$. The onset of increasing $P$ occurs at a $\phi$ where $Z$ begins its rapid increase. In fact, neither $P$ nor $Z$ are identically zero below jamming. Insight into this behavior follows from studies of shear carried out at $\phi$ 's below jamming, which we discuss in the next subsection.

We compare these experimental results to predictions above $\phi_{c}$ by means of least squares fits of $Z-Z_{c}$ and $P-P_{c}$ to $\phi-\phi_{c}$, as in Fig. 7. Necessarily, fit values for $\beta$ and $\psi$ depend on the choice of $\phi_{c}$ and whether rattlers are excluded or not. And, there is some ambiguity, due to the rounding in both quantities. Using several different approaches, described more fully in Majmudar et al., ${ }^{16}$ we obtain $\phi_{c}$ in the range 0.840 to 0.843 . In the former case, we obtain, $0.494 \leq \beta \leq 0.564$, and for the latter case, $0.363 \leq \beta \leq$ 0.525 . Overall, a logical choice for $\phi_{c}$ is $\phi_{c}=0.84220$ where $P$ rises above the background level. This choice, which corresponds to the results in Fig. 7, yields a consistent fit for both $P$ and $Z$. On balance, we, find that our values of $\beta \approx 0.55$ for the data without rattlers are larger than the value of 0.5 reported in, ${ }^{17,19}$ but smaller than those of Donev et al. ${ }^{20}$ who found 0.6 in 3D.

Fig. 7 also shows the variation of $P$ with $\phi$ (lower panel). These results indicate a clear transition at $\phi_{c}=0.8422 \pm 0.0005$. Least squares fitting $P-P_{c}$ above $\phi_{c}$ to $P-P_{c} \propto\left(\phi-\phi_{c}\right)^{\psi}$ yields $\psi=1.1 \pm 0.05$ for this choice of $\phi_{c}$. (Here, $P_{c}$ corresponds to the background $P$.) This exponent is consistent with the measured interparticle interaction force, and the expected exponent based on the simulations of Silbert and O'Hern et al. ${ }^{17,19}$ More detailed discussion is available in Majmudar et al. ${ }^{16}$

Finally, we consider the predictions of Henkes et al., ${ }^{3}$ by parametrically plotting the data of Fig. 7 in the form $P-P_{c} v s . Z$ above $\phi_{c}$. We remove the background pressure $P_{c}$ at $\phi_{c}$ (which we believe is due to induced shear strains). In this fit, we also normalize $P$ by $P_{c}$. These authors' predictions (which, as noted above, are a first attempt at constructing a field theory of the

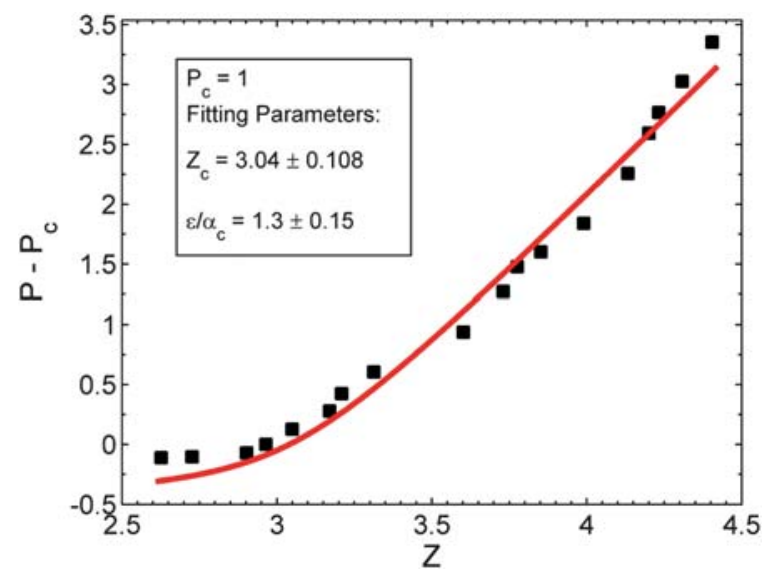

Fig. 8 Experimental data for $P$ vs. $Z$ (symbols) and a fit to the model of Henkes and Chakraborty ${ }^{3}$ (solid line). 
transition at Point $\mathrm{J})$ can be cast as $\left(P-P_{c}\right) / P_{c}=u-\left[\left(4 u^{2}+1\right)^{1 / 2}-\right.$ 1]/2, where $u=C\left(Z-Z_{c}\right) . C=\epsilon / \alpha_{c}$ is a system-dependent constant, where $\epsilon$ characterizes the grain elasticity, and $\alpha_{c}$ is the critical value for their generalized Boltzmann factor, $\alpha$. We have fitted to this form where $P_{c}, Z_{c}$, and $C$ are sensible fitting parameters. Fig. 8 shows reasonable, although not perfect, agreement with this prediction. From this fit, we obtain $Z_{c}=$ 3.04 , which is close to the nominal minimum isostatic value $Z_{c}=$ 3 , and is consistent with the analysis above.

The present experiments are consistent with predictions on many, but not all, fronts. We find a rapid, but not totally sharp increase in $Z$ at $\phi_{c}$. We also find power-law behavior above $\phi_{c}$ for $Z$ and $P$ with exponents $\beta \simeq 1 / 2$ and an exponent for $P$ of $\psi \simeq$ 1.1. These exponents are in good agreement with simulations. We also find reasonable agreement with the Henkes et al. theory. We note that the simulations and theory in question are for frictionless particles. Thus, agreement here is interesting, but not necessarily completely expected. There are some additional departures from the theory/MD pictures noted above. In addition, to the rounded transition in $Z$ near $\phi_{c}$, the amplitude of $Z$ $Z_{c} v s \phi-\phi_{c}$ differs from simulations. A comment on the presence of a background pressure is also relevant here. It is conceivable that this arises because the particles experience frictional forces with the base on which they rest. In fact, Majmudar et al. ${ }^{16}$ present analysis indicating the base friction is well below the level needed to produce the observe background pressure. Rather, we believe that this is due to small shear-induced stresses that are very difficult to remove in physical experiments. The discussion below on shear-induced jamming provides some additional insight into the role played by shear.

\subsection{Jamming under shear: anisotropic case}

We now turn to results for pure shear, as typified by Fig. 9. As noted in the experimental section, the initial state of this system is unjammed, i.e., $\phi=0.795$ is below the value for isotropic jamming. When shear is applied at constant density, a strong force chain network evolves, and for shear strains of a few percent, the system reaches a jammed state. Associated with jamming is the obvious formation of an oriented force chain network, as seen in the photoelastic images. The presence of this network is also manifested in the fabric, i.e. a purely geometric measure of inter-grain contacts. In Zhang et al. ${ }^{12}$ we show that statistically, the principal eigendirection of the fabric tensor aligns with the compressive direction at roughly the same time that the force chain network makes its appearance. In Fig. 9, we show the results of applying shear strains of up to $27 \%$, followed by a return to the original system geometry, all at fixed $\phi$. This corresponds to one half of a full shear cycle. Note that the final state, which corresponds to zero global strain, is jammed and contains a strong force network. We show data for a complete shear cycle, in Fig. 10, 11, and 12. These correspond to a shear strain to a maximum value, $\epsilon_{\max } \simeq 0.29$ from the initial state, followed by a shear strain reversal back to the original square shape of the boundaries, then a negative shear strain to $\epsilon \simeq 0.16$, and a return once again to the original boundary configuration. This particular shear cycle was the second in a series of six cycles. Hence, the data for $Z$ start at a value of well above 3.0. Similarly, the data for $P$ and $\tau$ start at values above 0. (In Zhang et al., ${ }^{12}$ we
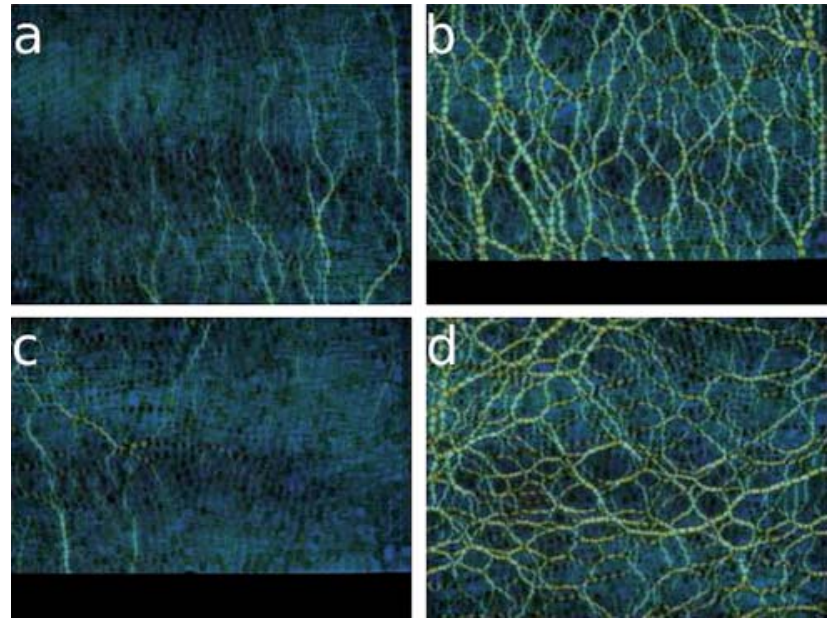

Fig. 9 Sequence of photoelastic images showing the evolution of the force chains as the system is sheared in the forward (a, b), and reverse direction (c, d). These four images are chosen at different steps from the 1 st shear cycle, out of a set of six complete cycles. The strains are $\epsilon=$ $0.033,0.267,0.267$, and 0.033 , for $\mathrm{a}, \mathrm{b}, \mathrm{c}, \mathrm{d}$, respectively. The original nearly stress-free state of (a) has the same density and boundary configuration as (d), which is jammed. In images b and c, the sidewall of the biax has moved into view, creating dark bands at the bottom of the images. Also, (c) was obtained following a small reverse strain step from (b).

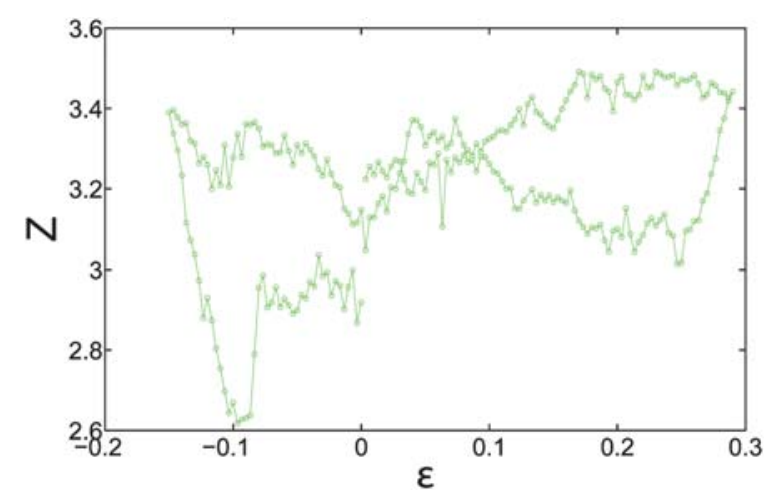

Fig. 10 Mean contact number $Z$ vs. strain $€$ for the second cycle.

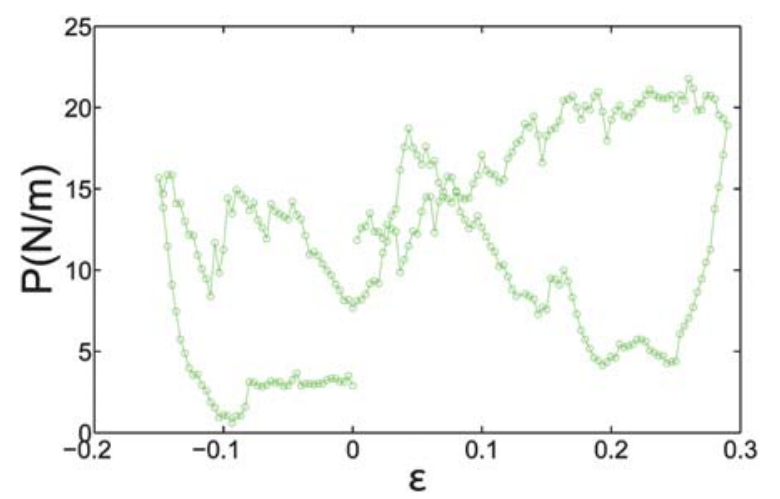

Fig. 11 Pressure, $P$ vs. strain, $\epsilon$, for the second cycle, showing strong hysteresis. 


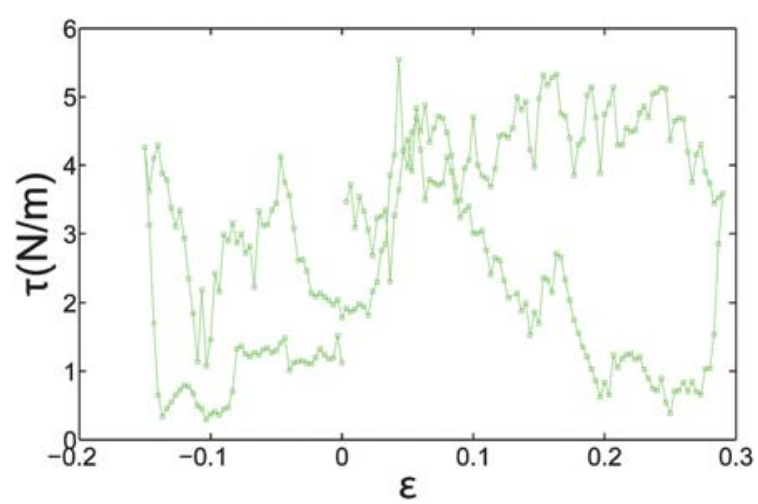

Fig. 12 Shear stress $\tau$ vs. strain, $\epsilon$ for the second cycle.

show results for the full six cycles.) These figures show unequivocally that the stresses and $Z$ are hysteretic in the strain. Particularly striking is the observation that the final state, part (d), of Fig. 9 is jammed, with a strong force network clearly visible, whereas the initial state before any shear strain was applied (a), was nearly unjammed. Again, we emphasize that the density is constant throughout. This means that neither the density nor the strain provide a unique characterization of a state. Hence, we must re-evaluate the nature of the jamming diagram when $\tau \neq 0$.

It is interesting that the hysteresis loops for all three of $Z$ and $P$ and $\tau v s . \epsilon$ are qualitatively similar, which suggests that there may exist a relation among them. The existence of such a relationship is demonstrated in Fig. 13 to 16, where we have accumulated data over six shear cycles, ${ }^{12}$ which are indicated by the various colors. In particular, results for $P v s$. $Z$ and $\tau v s$. $Z$, respectively, fall on nearly common curves.

There are several aspects of these figures that deserve discussion. In the first two of these figures, there is clearly rounding. This is due to several causes. These data have not been corrected for weak contacts, as discussed above. This correction is substantive, i.e. $\sim 15 \%$, when $\mathrm{Z} \lesssim 3.0$. This effect is within the noise of the data for larger $Z$. The effect of missed contacts on the

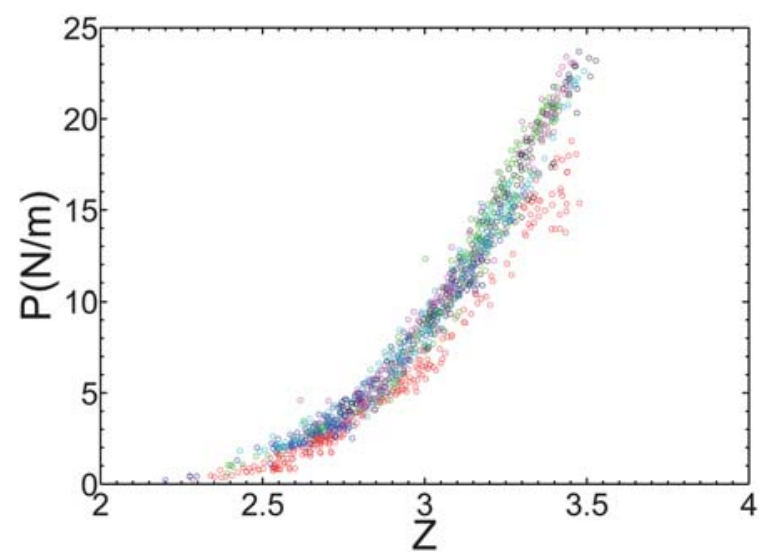

Fig. 13 Pressure, $P$, vs. average contact number, $Z$, without corrections for weak contacts. Different colors correspond to data from different shear cycles. Data points from the first shear cycle, shown in red, deviate slightly from other shear cycles.

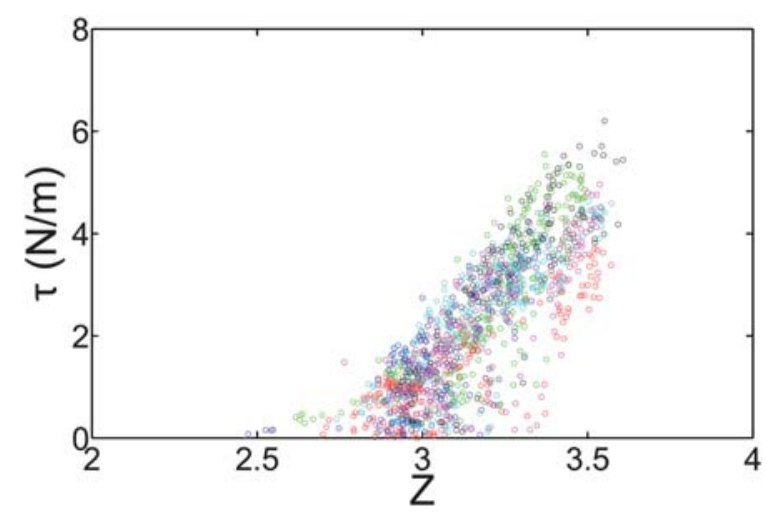

Fig. 14 Shear stress, $\tau$ vs. average contact number, $Z$.

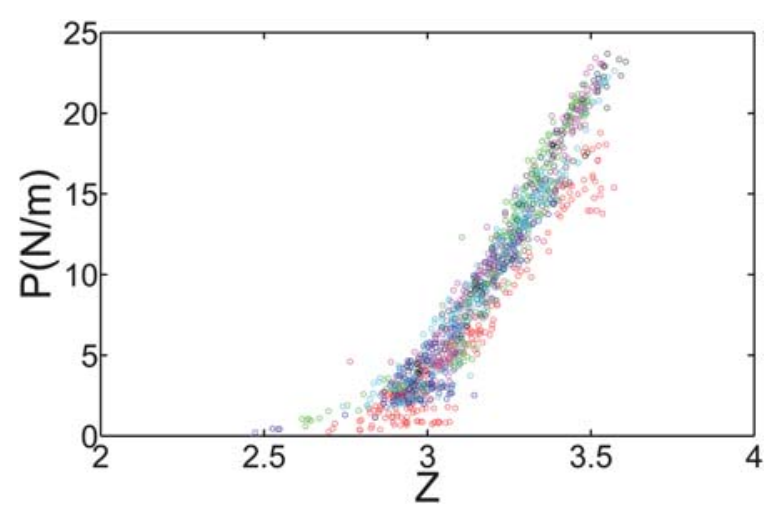

Fig. 15 Pressure, $P$, vs. average contact number, $Z$. Same data as the previous figure for $P$, but with a correction applied for weak contacts. Different colors correspond to data from the same shear cycles as above.

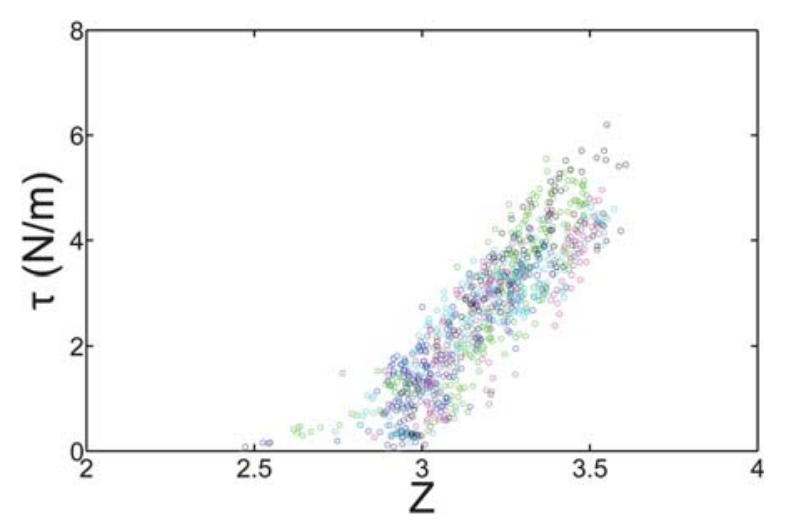

Fig. 16 Shear stress, $\tau v s$. average contact number, $Z$ after removal of data points from the first shear cycle and data points where the strong network direction is reforming.

pressure is much weaker than on $Z$. For $\tau v s$. $Z$, the relative scatter is higher than for $P$ vs. $Z$. A significant cause for the apparent scatter is the fact that during the first of the six shear cycles, the system exhibits transient behavior. Also, in relatively small ranges of strain following a reversal, the stresses and $Z$ depart from mean behavior, until a new network is formed. If we remove data from the first cycle and immediately after reversals, and correct for missed contacts, the results for $\tau$ vs. $Z$ yield 
a collapse that is comparable to that for $P$ vs. $Z$ as seen in Fig. 16 . We note that although $P$ and $Z$ do collapse, the spread of the data points around the curve is larger than the experimental errors. In fact, every time a new network of force chains is formed, following a shear reversal, there are small systematic differences in the stresses. The width of the scatter in these figures is primarily caused by these differences. Note that except for the switching regimes, the mean of the ratio $\tau / P$ is constant.

We conclude this section by considering the distributions of contact forces, as shown in Fig. 17 for the normal forces and in Fig. 18 for the tangential forces. In these distributions, we have normalized the data for $F_{n}$ or $F_{t}$ after each step by $\left\langle F_{n}\right\rangle$, the mean normal force at that strain step. We have combined the data for several adjacent steps within a cycle, but binned them separately for each shear cycle number. Specifically, ten for most data points and three to five for data near a reversal, are combined for each data point on the plots. We note that in general, distributions of $F_{n} /\left\langle F_{n}\right\rangle$ or $F_{t} /\left\langle F_{n}\right\rangle$ for single strain steps within a given shear cycle yield the same type of collapse, although the data are noisier.

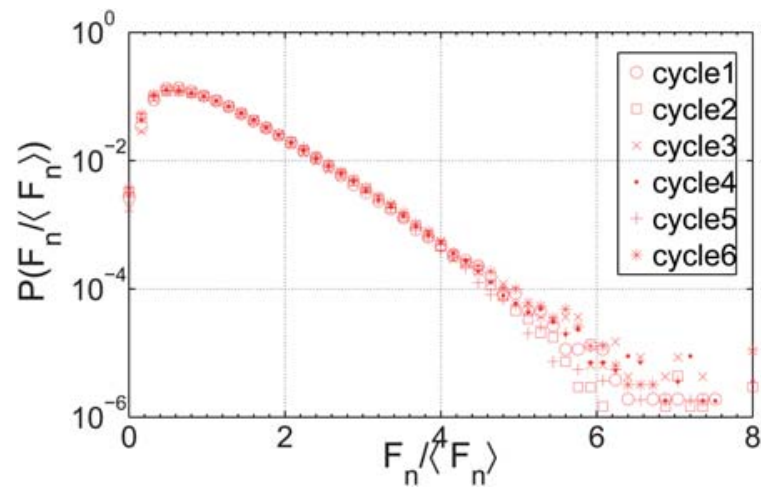

Fig. 17 Data for the distribution of normal forces, $F_{n}$, expressed as $F_{n} l$ $\left\langle F_{n}\right\rangle$ of all six shear cycles. We show data from all strain steps collectively for each cycle. Here, data for $F_{n} /\left\langle F_{n}\right\rangle$ are normalized by $\left\langle F_{n}\right\rangle$ for the given step.

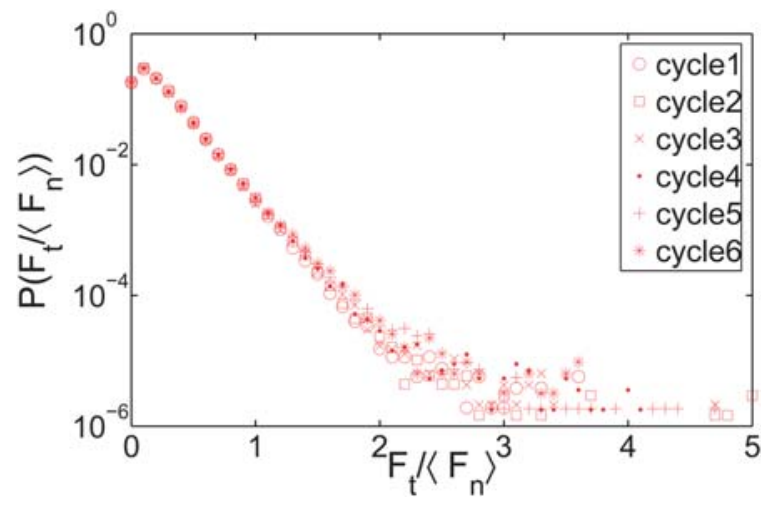

Fig. 18 Data for the distribution of normalized tangential forces, $F_{t} /\left\langle F_{n}\right\rangle$ of all six shear cycles. As for the distribution of normal forces, the statistics are combined for all steps within each cycle, where the normalization, $\left\langle F_{n}\right\rangle$ is made for each step.
A key point is that the distributions show a common form for all data of a given force type (i.e. normal vs. tangential forces). For normal forces, the distributions consist of a nearly exponential fall-off at large $F_{n} /\left\langle F_{n}\right\rangle$ and a peak at low $F_{n} /\left\langle F_{n}\right\rangle . P\left(F_{t} /\right.$ $\left.\left\langle F_{n}\right\rangle\right)$ also shows an exponential decay, but a weaker peak near 0 . Although some of the fall-off of both distributions at low force is due to the experimental lower limit of force detection, we believe that this is a relatively minor effect. The tangential force distributions, $P\left(F_{t} /\left\langle F_{n}\right\rangle\right)$, decay faster than that for the normal forces, which is due simply to the choice of normalization by $\left\langle F_{n}\right\rangle$ rather than by $\left\langle F_{t}\right\rangle$.

\section{Concluding remarks}

In this work, we have contrasted novel measurements of granular properties near jamming. These experiments are unique, to our knowledge, in their capacity to provide detailed grain-scale information on granular systems. These experiments are also highly novel because they have probed jamming behavior of anisotropic states.

We have sought to compare these experiments to theories of several types for the jamming transition: global scenarios, after Liu and Nagel, ${ }^{13}$ simulations, and statistical models..$^{3,417-20,24}$ At the most qualitative level, we have explored the paths in state space that granular systems follow for two contrasting cases: isotropic strain, and pure shear strain. The first case has produced experimental results that resemble several of the theoretical results discussed above, even though these typically apply to frictionless particles. In particular, we see jamming over a narrow range of packing fractions, and exponents for $Z$ and $P$ (but not necessarily amplitudes) that are consistent with frictionless models. And, we find reasonable consistency with the work of Henkes et al. . $^{3,4}$

The second, anisotropic, case yields results that deviate substantially from the usual jamming scenario. Specifically, we find that for $\phi$ 's which have unjammed states under isotropic conditions, it is possible to generate anisotropic jammed states by applying pure shear strain. The evolution of jammed states under shear strain is a manifestation of the same principle as Reynolds dilatancy. In his classic experiments, ${ }^{26}$ an elastic bag filled with granular material was subject to shear strain, with the result that the material expanded/dilated against the compliant container. In the present case, the boundaries do not allow for expansion during shear strain, and consequently, the pressure and shear stress grow. It is also interesting that anisotropic sheared states occur at the end of a shear cycle, when the boundaries of the system have been returned to the initial unstrained conditions. It seems likely that this last point is important for our studies of nominally isotropically deformed states. In particular, any small induced shear associated with the motion of the biax boundaries may lead to shear-induced stresses and hence, the observed background pressures and rounding in $Z$ observed for the isotropic jamming experiments.

The existence of jammed states below $\phi_{c}$ for isotropic jamming necessitates a new look at the jamming diagram, and we sketch a possibility that is consistent with the present experiments in Fig. 19. In part (a) of this figure, we sketch nominal paths for different packing fractions, projected onto the $\tau-P$ plane (light solid curves labelled 1 and 2). The dashed line corresponds to the 

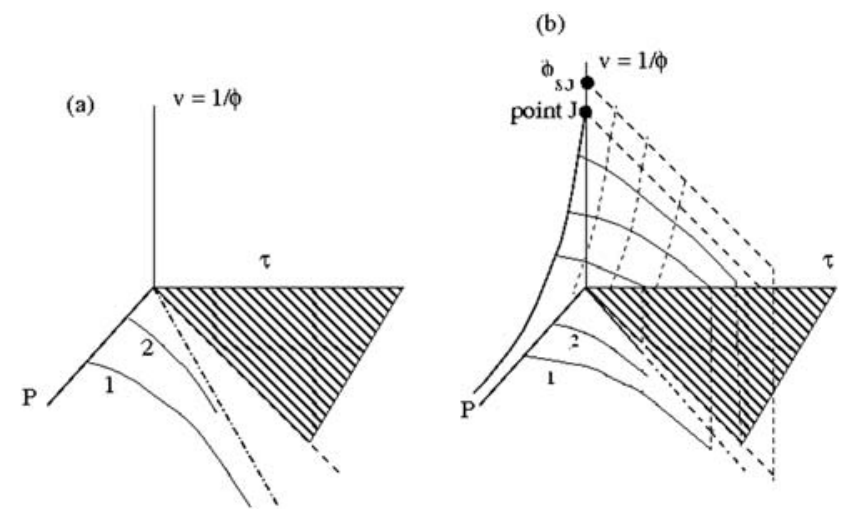

Fig. 19 Sketch of boundaries for jammed states. (a) Sketch of projections of special paths (see text) onto the $\tau-P$ plane. The curves labelled 1 and 2 are curves of constant density; the dot-dashed line is a nominal Coulomb line; and the dashed line is the limiting line $P=\tau$. (b) A rendering of these paths in the full $\tau-P-\phi$ plane.

formal limit where $P=\tau$. Since $P=\left(\sigma_{1}+\sigma_{2}\right) / 2$, and $\tau=\left(\sigma_{2}-\sigma_{1}\right) /$ 2 (taken here to be always positive), it is not possible to have a state where $\tau>P$, as long as the material is noncohesive, since the $\sigma_{i}$ are both positive (or zero). The disallowed region of the $\tau-$ $P$ plane is crosshatched out. In the present experiments, there exist states for some range $\phi_{S J}<\phi<\phi_{c}$ where it is possible to generate shear-jammed states. These states arise out of $P=\tau=0$, and to reasonable approximation, lie along a line $\tau=\mu P$, where $\mu<1$. Such a locus is indicated by the dashed-dot line in the $\tau-P$ plane. Although not discussed here in detail, we have also carried out pure shear, again at constant $\phi$, but this time with $\phi>\phi_{c}$, i.e. starting from isotropically compressed states. Necessarily, in these cases, the densities are above that for isotropic jamming, the initial pressure is positive, and the initial $\tau$ is zero. In such cases, $\tau$ starts at 0 , but grows as the system is strained. However, $P$ also grows under such strains, and the paths labeled ' 1 ' and ' 2 ' are hypothetical examples of such shear protocols, projected onto the $\tau-P$ plane. Under sufficient shear strain, individual contacts (and sometimes as a consequence multiple contacts) fail. However, it is worth remarking that after such a failure, the system typically finds a new jammed state, at somewhat reduced stress. Thus, there is always available a 'nearby' jammed state. From the Coulomb point of view, shear strain in these experiments leads to states that live on or close to the Coulomb cone. The application of shear at constant $\phi>\phi_{c}$ leads to relatively localized failures that reduce $\tau$ and maintain the system in the jammed region. In this sense, the present experiments indicate that for granular materials, the boundary in the $\phi-\tau$ plane when $\phi>\phi_{c}$ is not so much a limit between states in and out of mechanical equilibrium, e.g. jammed $v$ s. unjammed. Rather, it is a limit which the system does not cross while maintaining a fixed density. It is presumably the presence of such states that allow nearly rate-independent continuous shear strain of granular samples at low shear rates and in geometries (e.g. Couette ${ }^{27}$ ) that allow unbounded shear strain. If we then fill in the third direction of this state diagram, we obtain something like the sketch of Fig. 19.

An important issue concerns the variables used in Fig. 19. One could sketch a jamming diagram in the usual variables, where there is a line segment between $\phi_{S J}$ and $\phi_{J}$, but that does not very well represent the fact that this region has multiple states. That is, below Point-J, there are intrinsically anisotropic definitively jammed states that arise under shear strain, as well as isotropic unjammed states. Alternatively, one could replace $1 / \phi$ with $1 / P$. This has some drawbacks too. First, the jamming point moves to infinity, and second, all states at zero $P$ are now squeezed into the same point. One might also look for other variables, such as $\tau / P$, but this is awkward near Point-J. We have chosen the representation in Fig. 19 because it does provide a means of distinguishing the various states in question. We conclude by noting that granular systems (in the literal sense of 'granular') may be special due to the presence of non-zero friction in intergranular forces. This conclusion is supported by the recent numerical studies of Huessinger and Barrat ${ }^{28}$ which do not yield the same shear-jammed states seen in the present experiments.

\section{References}

1 S. F. Edwards and R. B. S. Oakeshott, Phys. D, 1989, 38, 88-92.

2 J. H. Snoeijer, T. J. H. Vlugt, M. van Hecke and W. van Saarloos, Phys. Rev. Lett., 2004, 92, 054302.

3 S. Henkes and B. Chakraborty, Phys. Rev. Lett., 2005, 95, 198002.

4 S. Henkes, C. S. O'Hern and B. Chakraborty, Phys. Rev. Lett., 2007, 99, 038002 .

5 T. Wakabayashi, J. Phys. Soc. Jpn., 1950, 5, 383-385.

6 P. Dantu, Proc. Of the 4th International Conf. On Soil Mech. and Foundation Eng., London, 1957, pp. 144-148.

7 A. Drescher and G. de Josselin de Jong, J. Mech. Phys. Solids, 1972, 20, 337.

8 T. S. Majmudar and R. P. Behringer, Nature, 2005, 435, 1079.

9 A. Smart and J. Ottino, Soft Matter, 2008, 4, 2125-2131.

10 R. P. Behringer, K. E. Daniels, T. S. Majmudar and M. Sperl, Philos. Trans. R. Soc. London, Ser. A, 2008, 366, 493-504.

11 A. Tordesillas, J. Zhang and R. P. Behringer, Geomechanics and Geoengineering, 2009, 4, 3.

12 J. Zhang, S. Majmudar, A. Tordesillas and R. Behringer, Granular Matter, 2010, 12, 159-172.

13 A. J. Liu and S. R. Nagel, Nature, 1998, 396, 21.

14 R. Jackson, Theory of Dispersed Multiphase Flow, Academic Press, New York, 1983, pp. 291-337.

15 C. A. Coulomb, Acad. R. Sci. Mem. Math. Phys. par Divers Savants, $1773,7,343$.

16 T. S. Majmudar, M. Sperl, S. Luding and R. P. Behringer, Phys. Rev. Lett., 2007, 98, 058001.

17 L. E. Silbert, D. Ertaz, G. S. Grest, T. C. Halsey and D. Levine, Phys. Rev. E: Stat., Nonlinear, Soft Matter Phys., 2002, 65, 031304.

18 C. S. O'Hern, L. E. Silbert, A. J. Liu and S. A. Langer, Phys. Rev. E: Stat., Nonlinear, Soft Matter Phys., 2003, 68, 011306.

19 C. S. O'Hern, S. A. Langer, A. J. Liu and S. R. Nagel, Phys. Rev. Lett., 2002, 88, 075507.

20 A. Donev, S. Torquato and F. H. Stillinger, Phys. Rev. E: Stat., Nonlinear, Soft Matter Phys., 2005, 71, 011105.

21 B. P. Tighe, J. E. S. Socolar, D. G. Schaeffer, W. G. Mitchener and M. L. Huber, Phys. Rev. E: Stat., Nonlinear, Soft Matter Phys., 2005, 72, 031306.

22 T. S. Majmudar, Ph.D. thesis, Duke University, Durham, NC, USA, 2006.

23 J. Zhang, R. P. Behringer and I. Goldhirsch, Progress in Theoretical Physics, 2010 accepted, to appear, 3-16.

24 M. Wyart, Phys. Rev. E: Stat., Nonlinear, Soft Matter Phys., 2005, 72, 051306.

25 S. Alexander, Phys. Rep., 1998, 296, 65-236.

26 O. Reynolds, Philos. Mag. Ser. 5, 1885, 50-20, 469.

27 D. Howell, R. P. Behringer and C. Veje, Phys. Rev. Lett., 1999, 82, 5241.

28 C. Huessinger and J.-L. Barrat, Phys. Rev. Lett., 2009, 102, 218303. 